\title{
Comparative study of organic and inorganic modification of Chinese fir wood based on the respiratory impregnation method
}

\author{
Ping $\mathrm{Li}^{\mathrm{a}} \mathrm{b}$, Yuan Zhang ${ }^{\mathrm{a}}$, Yingfeng $\mathrm{Zuo}^{\mathrm{a}^{*}}$, Jianxiong $\mathrm{Lu}^{\mathrm{c}}$, Guangming Yuan ${ }^{\mathrm{a}}$, Yiqiang $\mathrm{Wu}^{\mathrm{a}^{*}}$
}

${ }^{a}$ College of Materials Science and Engineering, Central South University of Forestry and Technology, Changsha, Hunan 410004, P.R. China

${ }^{\mathrm{b}}$ College of Art and Design, Xiangnan University, Chenzhou, Hunan 423000, P.R. China

${ }^{\mathrm{c}}$ Research Institute of Wood Industry, Chinese Academy of Forestry, Beijing 100091, P.R. China *Corresponding author

Yingfeng Zuo (Y. Zuo) and Yiqiang Wu (Y. Wu)

Affiliation: College of Materials Science and Engineering, Central South University of Forestry and Technology, Changsha, Hunan 410004, PR China

Email address: zuoyf1986@163.com (Y. Zuo) and wuyq0506@126.com (Y.Wu)

Phone number: +86 73185623898

Fax: +8673185623898

Abstract: To compare The effects of organic and inorganic impregnation on the properties of unmodified, phenol formaldehyde oligomer-modified (PFOMCF), and sodium silicate-modified Chinese fir wood (SSMCF) were compared using samples prepared using the respiratory impregnation method. Impregnation and reinforcement effects and water resistance of PFOMCF and SSMCF were compared and the results was showed that the weight percentage gain, density increase rate, bending strength, and compressive strength of SSMCF were clearly higher than those of PFOMCF and had a lower water absorption rate within $60 \mathrm{~h}$. The impregnation and reinforcement effects and dimensional stability of SSMCF were better than those of PFOMCF. FT-IR, XRD, CONE, and TGA examinations were used to test and analyze the chemical structure, crystalline structure, flame retardancy, and heat resistance of these modified woods. The results indicated that SSMCF possessed more hydrogen bonds than PFOMCF and that $\mathrm{Si}-\mathrm{O}-\mathrm{Si}$ chemical bonding with high bond energy was formed. Meanwhile, the weakened degree of the diffraction peak of SSMCF was much less than that of PFOMCF. These results explained that the mechanical properties and water resistance of SSMCF were better than PFOMCF. Compared with PFOMCF, SSMCF had a lower heat release rate (HRR), peak-HRR, mean-HRR, total heat release, smoke production rate, and total smoke production as well as higher thermal decomposition temperature and residual rate. Inorganic sodium silicate was shown to be a better flame retardant, while SSMCF had good smoke suppression effects, thermal stability, and safety performance in the case of fire.

Keywords: Chinese fir wood; Sodium silicate; Phenol formaldehyde oligomer; Respiratory impregnation; Comparative study

\section{Introduction}

Chinese fir (C. lanceolate) is one of the most important plantation tree species in China [1]. 
Its planting area accounts for $\sim 25 \%$ of the total Chinese plantation area because of its rapid growth, straight texture, few pests and diseases, and easy processing [2,3]. However, Chinese fir wood has some shortcomings, such as low density, short fibers, high $\mathrm{pH}$ value, severe cracking and deformation, high moisture content, low strength, poor stiffness, and large chord-radial shrinkage ratio. These shortcomings render Chinese fir products easy to deform and crack in use and greatly limits its applications in furniture, floors, wood structures, and other building materials [4]. Therefore, it is increasingly important to strengthen and dimensionally stabilize this wood. Injecting organic resin or an inorganic material into Chinese fir wood and curing it can improve its strength, corrosion resistance, wear resistance, water resistance, weather resistance, dimensional stability, and subsequent processing [5,6]. After impregnation modification, the physical and mechanical properties of the wood have been significantly improved [7], such that it can replace high-quality natural forest trees and be used as structural materials, such as in brackets, trusses, carriage floors, and container boards, thus significantly improving the wood's applications and value.

Previous studies have focused on impregnation modification of Chinese fir wood with organic resin, especially an aldehyde resin. Aldehyde resins not only play a filling role in wood modification but also react with the wood's active functional groups to form a new stable structure, improving the wood's corrosion resistance, water resistance, dimensional stability, and physical and mechanical properties [8,9]. However, impregnation of organic resin has a negative impact on wood finishing and bonding properties and the price of these organic chemicals is high. More unacceptable in this impregnation process is the release of some toxic gases, which pose threats to the environment and human safety [10]. In contrast, impregnation with inorganic substances has no adverse effect on wood finishing and gluing, is inexpensive, natural, and nontoxic [11] and the visual environmental characteristics of wood are retained to a great extent. Moreover, inorganic modifiers have the advantages of low cost and being environmentally-friendly, and inorganic-modified Chinese fir wood effectively has improved mechanical strength, anti-corrosion, and flame retardancy $[12,13]$. Inorganic modified Chinese fir wood has wide application prospects in furniture manufacturing and interior decoration, which are in direct contact with people. Therefore, inorganic-reinforced Chinese fir wood has gradually become a focus of current research.

The differences between inorganic and organic impregnation modifications on Chinese fir wood properties were examined by comparing phenol formaldehyde oligomer-modified (PFOMCF) and sodium silicate-modified Chinese fir wood (SSMCF), prepared by the respiratory impregnation method. While phenol formaldehyde oligomer is the most commonly used organic modifier and thus most widely studied, sodium silicate is an inorganic modifier with environmental protection, flame retardancy, and low cost. The respiratory impregnation method is a method that simulates the biological respiratory process, alternating positive and negative pressure circulation. Through negative pressure treatment of the wood, the internal pores and cell 
cavities are fully compressed. When the negative pressure is converted to positive pressure, the pores and cavities are fully opened, forming channels for the entry of modifiers and significantly improving impregnation. The differences of the impregnation and reinforcement effects and water resistance between PFOMCF and SSMCF were analyzed by comparing its weight percentage gain, density increase ratio, blending strength, compressive strength, hardness (end face, diameter, and chord face), and water absorption rate. Fourier-transform-infrared and X-ray diffraction spectroscopies, cone calorimetric analysis, and thermogravimetric analyses (FT-IR, XRD, CONE, and TGA, respectively) were used to test and analyze the chemical structure, crystalline structure, flame retardancy, and heat resistance of modified wood. The purpose was to improve the wood properties and resulting product, providing added value to Chinese fir plantations and expanding their scope of application.

\section{Materials and Methods}

\subsection{Materials}

Chinese fir wood used in this experiment was cut from Yongzhou, Hunan Province, China. Test specimens were prepared according to the Chinese standard GB/T1929-2009. Six samples of $20 \times 20 \times 20 \mathrm{~mm}$ (tangential $\times$ radial $\times$ longitudinal) were prepared for weight percentage gain and density tests. Twelve samples of $300 \times 20 \times 20 \mathrm{~mm}$ were produced for bending strength testing and 12 samples of $50 \times 50 \times 70 \mathrm{~mm}$ produced for compressive strength testing. Specimens used had no knots, cracks, decay, oblique texture, or other defects. Sodium silicate (44.5\%, 3.4 modulus) was purchased from Hunan Hetang Chemical Co., Ltd. (Changsha, Hunan, China). Formaldehyde (37.0\% aqueous solution, AR), phenol (AR), and sodium hydroxide (AR) were obtained from Sinopharm Chemical Reagent Co., Ltd. (Shanghai, China). Ultrapure water was prepared using a ZWL-PA1-20 ultrapure water system (Hunan Zhongwo Water Environmental Protection Technology Co., Ltd., Hunan, China).

\subsection{Preparation of phenol formaldehyde oligomer [14]}

Phenol formaldehyde oligomer (PFO) was prepared with a molar ratio of phenol/formaldehyde/sodium hydroxide as 1/3.5/1. Formaldehyde and phenol were accurately weighed and poured into four bottles with an agitator and placed in a water bath adjusted to $30^{\circ} \mathrm{C}$. The sodium hydroxide was mixed with ultrapure water to form a $50 \mathrm{wt}-\%$ sodium hydroxide aqueous solution, which was then slowly added into four bottles within $30 \mathrm{~min}$. After $40 \mathrm{~h}$ of reaction, the PFO was discharged. The main components of the PFO were 2,4-dihydroxymethyl phenol, 2,6-dihydroxyphenol, and 2,4,6-trihydroxyphenol (Fig.1), with the solid content at 43.5\% and viscosity of $52.2 \mathrm{mPa} / \mathrm{s}$.

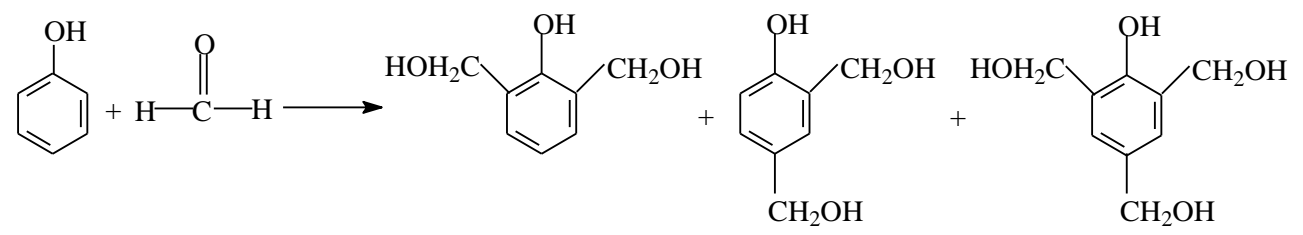

Fig.1 Reaction principle of phenol formaldehyde oligomer 


\subsection{Preparation of modified Chinese fir wood}

Chinese fir wood specimens for density, hardness, bending, and compression testing were selected and dried in an electric heat blast-drying chamber at $60^{\circ} \mathrm{C}$ until the moisture content ranged from 10 to $12 \%$. The specimens were impregnated in an impregnation tank by the respiratory impregnation method, the principle of which is illustrated in Fig.2. First, the impregnating tank was evacuated to $-0.1 \mathrm{MPa}$ and pressure held for $10 \mathrm{~min}$. Organic sodium silicate or inorganic PFO modifiers was mixed into a solution at $20 \mathrm{wt} \%$, sucked into the impregnating tank by the negative pressure, and the pressure then released. Second, the impregnation system was pressurized by an air compressor to $0.5 \mathrm{MPa}$ for $20 \mathrm{~min}$. The organic or inorganic modifiers was then unloaded and discharged from the impregnating tank. Four cycles were performed with this method with alternating positive-negative pressure and the total immersion time of $2 \mathrm{~h}$. The pressure was finally relieved, the impregnated wood removed, and the surfaces washed and dried. The samples were dried at $120^{\circ} \mathrm{C}$ for $6 \mathrm{~h}$ to obtain the sodium silicate-modified (SSMCF) or phenol formaldehyde oligomer-modified Chinese fir wood (PFOMCF).

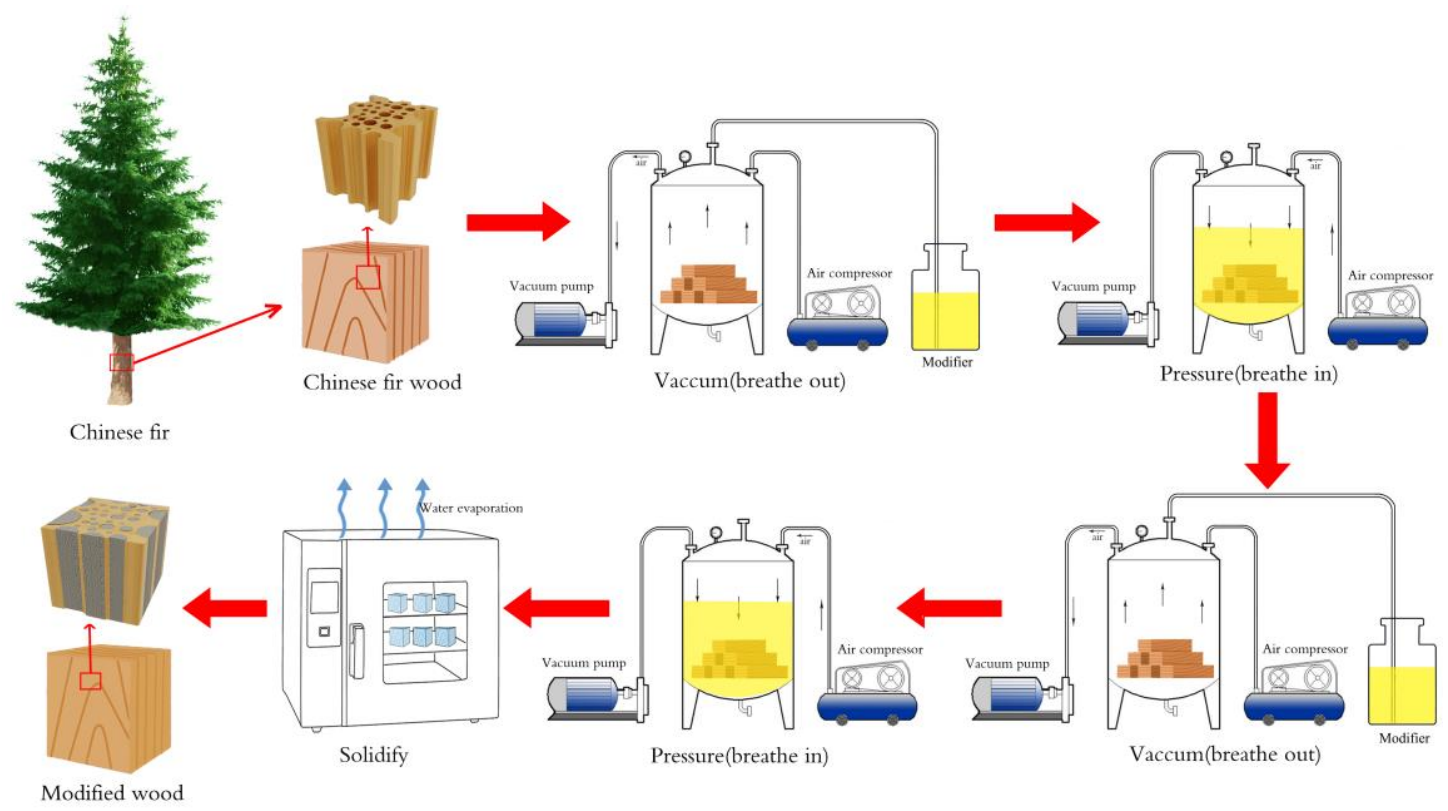

Fig.2 Respiratory impregnation modification process of Chinese fir wood

\subsection{Properties and characterization}

\subsubsection{Weight percentage gain}

The oven-dried weight of Chinese fir specimens before and after impregnation was recorded as $m_{0}$ and $m_{1}$, respectively. The weight percentage gain (WPG) was assessed using Eq.(1).

$$
\mathrm{WPG}=\left(m_{1}-m_{0}\right) / m_{0} \times 100 \%
$$

\subsubsection{Density increase ratio}

Referring to GB/T 1933-2009, Wood density measurement method, for testing, the wood was dried to absolute dryness in a blast oven at $103 \pm 1{ }^{\circ} \mathrm{C}$ and recorded as $m_{0}$. The longitudinal $L_{0}$, the radial $R_{0}$, and the tangential $T_{0}$ were assessed using a vernier caliper. The density of the specimen 
before impregnation was calculated as $\rho_{0}=\left[m_{0} /\left(L_{0} \times \mathrm{R}_{0} \times \mathrm{T}_{0}\right]\right.$. The immersion modification of Chinese fir specimens was assessed as $m_{1}$ and the longitudinal $L_{1}$, radial $R_{1}$, and tangential $T_{1}$ measured. In addition, the density of the specimens after immersion was calculated as $\rho_{1}=\left[m_{1} /\right.$ $\left(L_{1} \times R_{1} \times T_{1}\right)$ ]. The density increase ratio $(\Delta \rho)$ was assessed using Eq. (2).

$$
\Delta \rho=\left(\rho_{1}-\rho_{0}\right) / \rho_{0}
$$

\subsubsection{Mechanical properties test}

Wood bending strength was tested in accordance with the Chinese national standard GB/T 1936-2009. The dimensions during the bending strength test were $300 \times 20 \times 20 \mathrm{~mm}$. The load was applied in the tangential direction of the samples, with a distance of $240 \mathrm{~mm}$ between two fulcrums. Twelve samples were tested in each experimental condition for both treated and control samples.

Compressive strength was tested according to the Chinese national standard GB/T 1935-2009. Specimens with a size of $20 \times 20 \times 30 \mathrm{~mm}$ were loaded at a constant loading rate and the maximum compressive load under which the sample stood for more than $90 \mathrm{~s}$ without collapse recorded.

The hardness values of the end face, radial face, and bastard face were measured with reference to Chinese national standard GB/T 1941-2009. Each specimen with the size of $70 \times 50 \times 50 \mathrm{~mm}$ was tested once at two end faces, two radial faces, and two bastard faces. The hemispherical steel indenter was pressed into the test surface at a uniform rate of $3-6 \mathrm{~mm} / \mathrm{min}$ until an indent depth of $5.64 \mathrm{~mm}$, with the load reading accurate to $10 \mathrm{~N}$.

\subsubsection{Water absorption rate}

The water absorption coefficient (WAR) was tested according to the Chinese national standard GB/T 1934.1-2009. Dry specimens were immersed in water at $20 \pm 5^{\circ} \mathrm{C}$. Specimens were taken out every $24 \mathrm{~h}$ and weighed after surface water was removed using a filter paper. The detection period was $60 \mathrm{~h}$, with the WAR calculated using Eq. (3).

$$
\mathrm{WAR}=\left(W_{2}-W_{1}\right) / W_{1} \times 100 \%
$$

where $W_{1}$ is the mass of oven-dried wood sample before impregnation and $W_{2}$ the mass of oven-dried wood sample after impregnation.

\subsubsection{Fourier transform infrared spectroscopy analysis}

Chinese fir wood samples were ground and crushed into small particles in a mortar with a diameter of $<0.074 \mathrm{~mm}$ (200-mesh). An IRAffinity-1 Fourier transform infrared (FTIR) spectrometer (Shimadzu Corp., Kyoto, Japan.) was used to test the particles. Samples were ground to powders with $\mathrm{KBr}$ in a weight ratio of $1 / 100$. FTIR curves of samples were obtained in the range of $400-4000 \mathrm{~cm}^{-1}$.

\subsubsection{X-ray diffraction analysis}

Samples were completely dried in a vacuum oven at $50^{\circ} \mathrm{C}$ for $48 \mathrm{~h}$ to remove remaining moisture. The crystallinity index of the samples was measured using an X-ray diffractometer (XD-2, Purkinje General Instrument Co., Ltd., Beijing, China) with a $\mathrm{Cu}$ target at $36 \mathrm{kV}$ and 20 
$\mathrm{mA}$. The samples were tested in the angular range of $2 \theta=5-40^{\circ}$ with a scanning rate of $4 \% \mathrm{~min}$. The empirical crystallization index Crl, proposed by Segal [15]. The calculation used to determine this parameter expressed as Eq. (4).

$$
C r l=\frac{I_{002}-I_{\text {amorph }}}{I_{002}} \times 100 \%
$$

where $I_{002}$ is the maximum diffraction peak intensity of the main crystallization peak 002 and $I_{\text {amorph }}$ the diffraction intensity of $2 \theta$ angles to $18^{\circ}$.

\subsubsection{Cone calorimetric}

CONE tests of the Chinese fir wood were performed on a cone calorimeter (FTT; Stanton Redcroft Inc., East Grinstead, UK) following ISO5660-1. For each test, 100×10×100 mm (T × R × L) specimens were covered with aluminum foil, except the upper surface, which was placed into a specific steel frame, the steel frame mounted horizontally on the loader, and then exposed to heat radiation of $50 \mathrm{~kW} / \mathrm{m}^{2}$. This heat radiation corresponded to a temperature of $780^{\circ} \mathrm{C}$ on the upper test sample surface.

\subsubsection{Thermo gravimetric analysis}

TGA measurements of the Chinese fir wood samples were conducted with 209 F3 TGA (Netzsch Instruments, Inc., Burlington, MA, USA). Approximately $5 \mathrm{mg}$ of dried sample were placed in a platinum crucible and heated from 25 to $600^{\circ} \mathrm{C}$ at the rate of $10^{\circ} \mathrm{C} / \mathrm{min}$, with a nitrogen gas flow rate at $30 \mathrm{~mL} / \mathrm{min}$. TGA and derivative thermogravimetric analysis (DTG) data of each sample were obtained.

\subsubsection{Statistical analysis}

Data were statistically evaluated using the statistical software package Minitab Version 15 and reported as the mean value \pm standard deviation of replicates. A single-factor analysis of variance was conducted to identify significant differences among mean values according to least significant difference criteria with a 95\% confidence level $(p<0.05)$.

\section{Results and Discussion}

\subsection{Comparison of impregnation effects}

The weight percentage gain and density increase ratio of impregnated Chinese fir wood directly reflected the impregnation effects on the wood. Therefore, the effects of organic and inorganic modifiers on the impregnation effect of the wood were evaluated by comparing the weight percentage gain and density increase ratios of PFOMCF and SSMCF. The results are shown in Fig.3.

The weight percentage gain and density increase ratio of SSMCF were $44.45 \pm 1.31$ and 45.14 $\pm 1.28 \%$ while those of PFOMCF were $52.20 \pm 1.88$ and $53.44 \pm 1.59 \%$, respectively (Fig. 3 ). The weight percentage gain and density increase ratio of SSMCF were clearly higher than those of PFOMCF, which indicated showed that the impregnation effect of SSMCF was better than that of PFOMCF. The experimental results showed that the densities of $3.4 \mathrm{M}$ sodium silicate solution and phenol formaldehyde oligomer were 1.42 and $1.20 \mathrm{~g} / \mathrm{cm}^{3}$, respectively. The solid content of 
the two modifiers was 44.5 and $43.5 \%$, indicating that the concentrations of the two modifiers were basically the same. This also indicated that the density of inorganic sodium silicate-soaked in Chinese fir wood was higher than that with organic phenol formaldehyde oligomer, which resulted in the weight percentage gain and density increase ratio of SSMCF being greater than those of PFOMCF. In addition, the molecular weight of sodium silicate was 122 , while that of hydroxymethyl, dihydroxymethyl, and trihydroxymethyl phenols in phenol formaldehyde oligomers were 124, 154, and 184, respectively. Because the molecular weight of sodium silicate was less than that of phenol formaldehyde oligomer, it more easily penetrated the wood's pores.

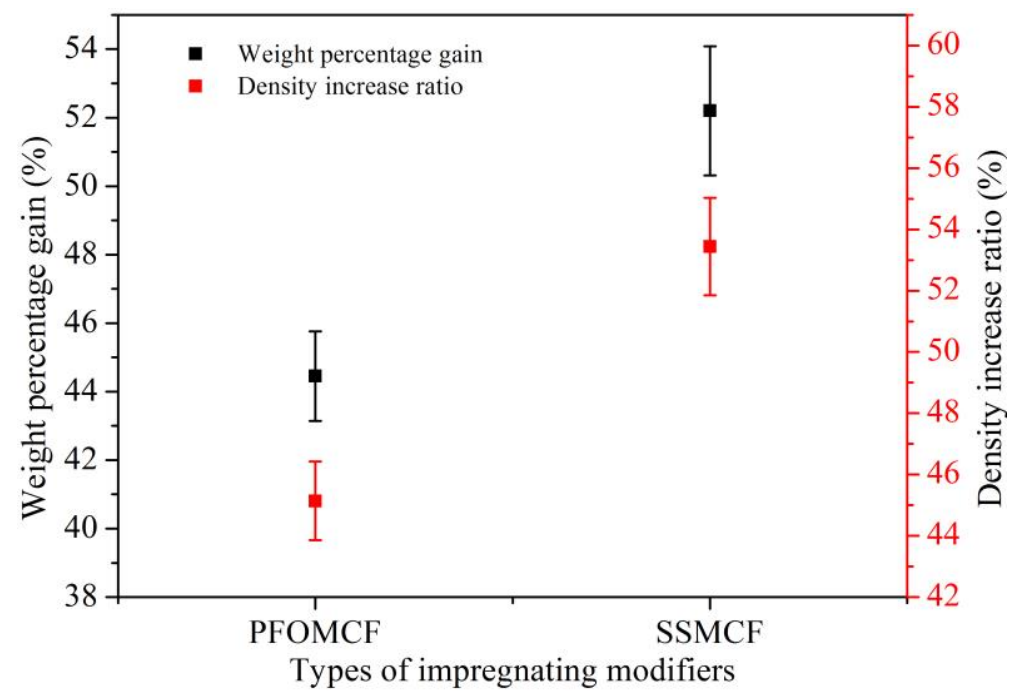

Fig.3 Weight percentage gain and density increase ratio of SSMCF and PFOMCF

\subsection{Comparison of strengthen effects}

There are some defects in Chinese fir wood, including loose material and low strength [16], and impregnating wood with a filler is an effective method of improving its mechanical properties. Therefore, the bending and compressive strengths and hardness are the most significant parameters for the utilization of Chinese fir wood. To compare the strengthening effects of this wood modified by inorganic sodium silicate and organic phenol formaldehyde oligomer, the bending strength, compressive strength, and hardness of SSMCF and PFOMCF were tested. The results are shown in Fig.4 and Fig.5.

The bending and compressive strengths of unmodified Chinese fir wood were $46.50 \pm 2.58$ and $22.54 \pm 1.97 \mathrm{MPa}$, respectively (Fig. 4). After impregnation modification, the bending and compressive strengths of SSMCF and PFOMCF were significantly improved. These results showed that impregnation modification effectively improved the wood strength, which was attributed to that the introduction of sodium silicate and phenol formaldehyde oligomer into the wood, which solidified in the wood cell cavities and cell wall spaces to form a hard, solid structure [17]. However, there were some differences in the strengthening effects between SSMCF and PFOMCF. The bending and compressive strengths of SSMCF were higher than those PFOMCF, which was directly related to the impregnation effect of the two modifiers. The better the impregnation effect, the more significant the strength improvement. At the same time, it was 
indicated here that the inorganic impregnation modification of sodium silicate had a better strengthening effects than modification with phenol formaldehyde oligomer.

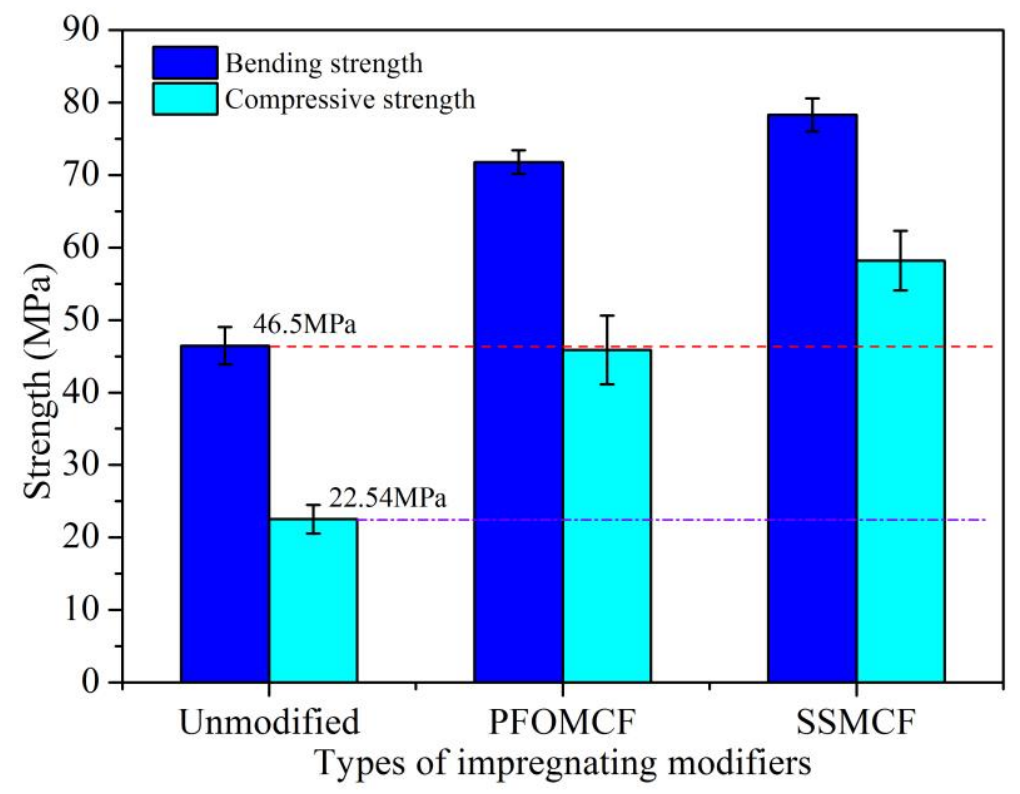

Fig.4 Bending strength and compressive strength of unmodified Chinese fir wood, PFOMCF and SSMCF

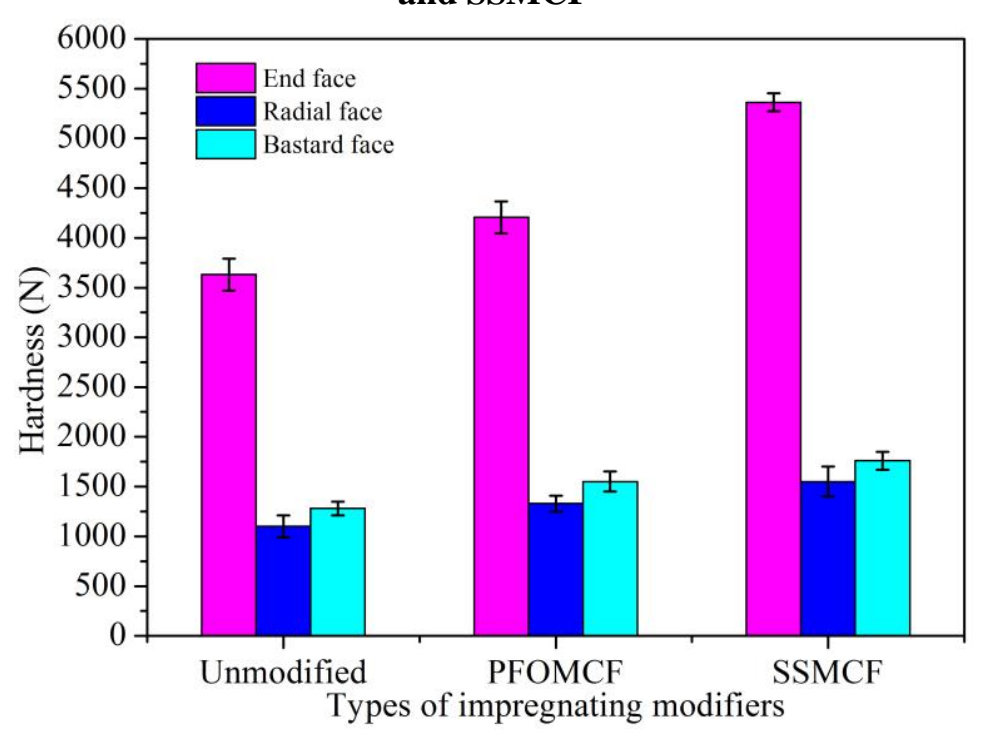

Fig.5 End face, radial face and bastard face hardness of unmodified Chinese fir wood, PFOMCF and SSMCF

Comparison with unmodified Chinese fir wood showed that the end, radial, and bastard face hardness of SSMCF and PFOMCF were significantly improved (Fig.5). The three-surface hardness of SSMCF was higher than that of PFOMCF, which showed that inorganic impregnation with sodium silicate was better than organic impregnation with phenol formaldehyde oligomer for reinforcement of this wood.

\subsection{Comparison of water resistance}

There are many pores in the wood structure, which include a large number of hydrophilic groups. Therefore, wood hygroscopicity occurs in a wet environment, which leads to deformation, 
cracking, and other defects. The effects of inorganic and organic impregnation modification on the water resistance of this wood were verified by examining the water absorption rate of unmodified Chinese fir wood, PFOMCF, and SSMCF over $60 \mathrm{~h}$. The results are shown in Fig.6.

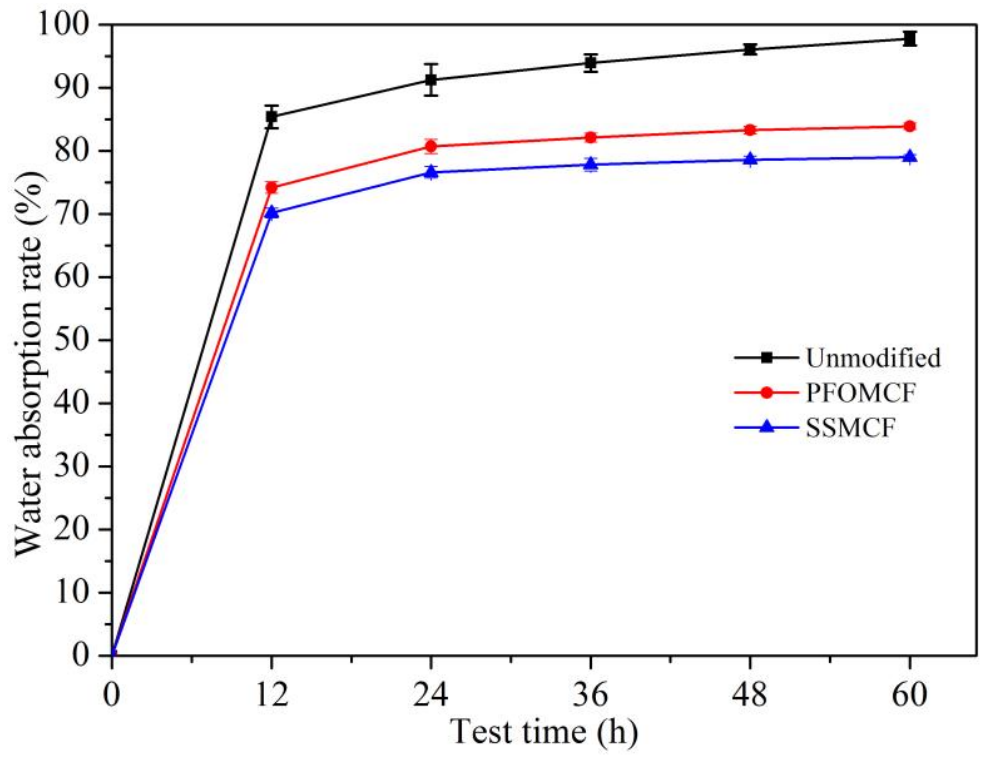

Fig.6 Water absorption rate of unmodified Chinese fir wood, PFOMCF and SSMCF

As can be seen from Fig.6, The water absorption rate of PFOMCF and SSMCF was lower than that of unmodified wood within the $60 \mathrm{~h}$ test time. The results showed that impregnation with silicate and phenol formaldehyde oligomer effectively improved wood water resistance, thus improving its dimensional stability. On one hand, most of the wood voids were filled, after impregnation with sodium silicate and phenol formaldehyde oligomer, which reduced the water storage area. On the other hand, the modifier immersed in the wood reacted with hydroxyl groups in the wood components to form stable chemical bonds and effectively reduced the number of hydrophilic groups. However, the water absorption rate of SSMCF was lower than that of PFOMCF within $60 \mathrm{~h}$, which was attributed to that the weight percentage gain and density increase ratio of SSMCF, which was greater than that of PFOMCF. This resulted in more silicates being introduced into the wood interior, filling of more pores, and formation of more chemical bonds. This not only reduced the water storage space in the wood, but also reduced the amount of water absorbent groups. These two effects made the water resistance of SSMCF better and more conducive to maintaining dimensional stability.

\subsection{Comparison of chemical structure}

Wood is a natural complex composed mainly of cellulose, hemicellulose, and lignin, which contain a large number of reactive groups, such as hydroxyl groups [18]. Also, sodium silicate ions, $\mathrm{SiO}_{3}{ }^{2-}$, and the molecular chains of phenol formaldehyde oligomer have greater numbers of reactive hydroxymethyl phenol groups. Therefore, sodium silicate and phenol formaldehyde oligomer can form hydrogen bonds and some chemical bonds with hydroxyl groups in wood. To verify the chemical bonding between impregnating modifiers and this wood, FT-IR was used to compare unmodified wood with PFOMCF and SSMCF. The results are illustrated in Fig.7. 


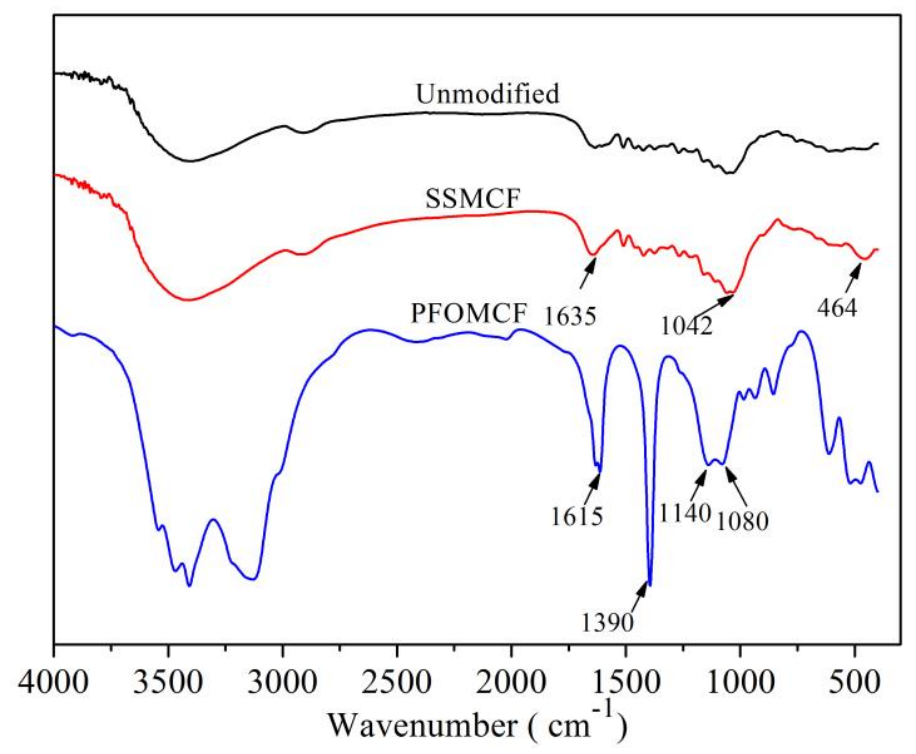

Fig.7 Infrared Spectra of unmodified Chinese fir wood, PFOMCF and SSMCF

In comparison with unmodified Chinese fir wood, the vibration peak bands of hydroxyl absorption of PFOMCF and SSMCF were widened and substantially strengthened in the range of $3000-3600 \mathrm{~cm}^{-1}$ (Fig.7). The results indicated that the content of free hydroxyl groups decreased and the associative hydroxyl groups increased in the wood. In addition, the absorption peak strengths of PFOMCF and SSMCF at other locations also increased. For SSMCF, vibration peaks of $\mathrm{Si}-\mathrm{O}-\mathrm{Si}$ appeared at 1,042 and $464 \mathrm{~cm}^{-1}$ and the vibration absorption peak of $-\mathrm{OH}$ stretching near 3,300 and 1,635 $\mathrm{cm}^{-1}$ increased [19]. This indicated that silicate was immersed in the wood and formed chemical bonds with wood hydroxyl groups and the content of associated hydroxyls increased. Silicates formed chemical and hydrogen bonds with hydroxyl groups in the wood, and rather than being a simple physical filling, this indicated that a large number of hydroxyl groups in the wood were sealed or bonded and greatly improved the mechanical properties and water resistance of modified wood impregnated with sodium silicate. For PFOMCF, the strength of $-\mathrm{C}=\mathrm{C}$ - stretching vibration peak on benzene ring was near $1615 \mathrm{~cm}^{-1}$, the methylene bending vibration peak near $1390 \mathrm{~cm}^{-1}$, and C-O stretching vibration peak on $-\mathrm{CH}_{2} \mathrm{OH}$ within $1080-1140$ $\mathrm{cm}^{-1}$ were significantly enhanced. It was concluded that phenol formaldehyde oligomer better infiltrated into the wood and formed certain ether bonds with hydroxyl groups in the wood. The mechanical properties and water resistance of PFOMCF were also improved to some extent. However, the impregnation effect of PFOMCF was not as good as SSMCF, and the bond energy of ether bonds was much lower than that of $\mathrm{Si}-\mathrm{O}-\mathrm{Si}$ bonds. This explained why PFOMCF was not as effective as SSMCF in strengthening and water resistance modifications.

\subsection{Comparison of crystal structure}

Cellulose is a very important component in wood chemical composition and cellulose molecular chains are aligned and ordered to produce crystalline regions [20]. The crystallinity of cellulose is closely related to the physical, mechanical, and chemical properties of wood. Therefore, to study the effects of inorganic and organic impregnation modification on the 
crystalline structure of Chinese fir wood, XRD recuts were used to test the unmodified wood, PFOMCF, and SSMCF. The results are presented in Fig.8.

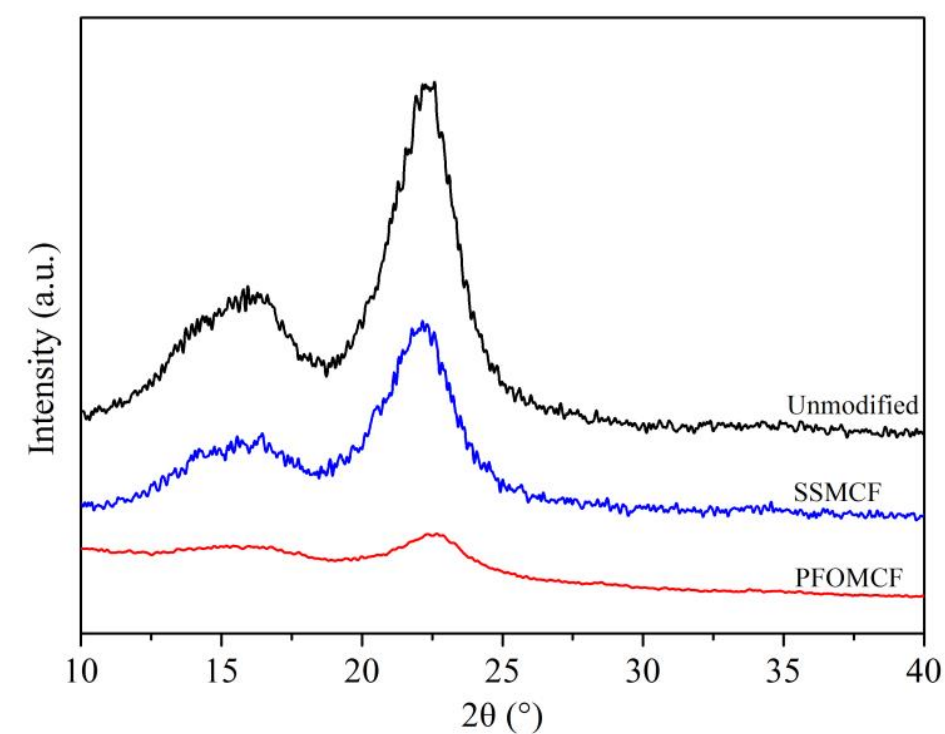

Fig.8 XRD diffraction patterns of unmodified Chinese fir wood, PFOMCF and SSMCF

It can be seen from Fig.8 that the diffraction peaks of wood cellulose at (101), (002), and (040) crystalline planes occurred in unmodified Chinese fir wood when $2 \theta$ was $16,22.5$, and $35^{\circ}$, respectively [21]. XRD peaks of wood modified by silicate and phenol formaldehyde oligomer did not shift, indicating that the unit cell types of modified Chinese fir wood did not change. However, it was clearly observed that the intensity of crystallization diffraction peaks of the two kinds of wood decreased to various degrees. The reasons for this change were because, ov the one hand, impregnating modifiers were immersed into the noncrystalline zone of the wood, which produced a certain swelling effect in the process of infiltration, wetting, diffusion, adsorption, and curing of wood cellulose. This series of processes disturbed well-ordered microfibrils in the crystallization zone and reduced the relative crystallinity. On the other hand, the impregnating modifiers infiltrated into the cellulose molecular chain and reacted with the wood hydroxyl groups, which destroyed cellulose hydrogen bonds and weakened intermolecular interactions between cellulose molecular chains. However, the lower diffraction peak of SSMCF was much less than that of PFOMCF, which showed that phenol formaldehyde oligomer decreased wood crystallinity to a greater extent. The mechanical strength of the wood was seriously affected by decreased crystallinity, which confirmed that the blending strength, compressive strength, and hardness of PFOMCF were lower than those of SSMCF. Meanwhile, the decreased crystallinity made the molecular chains of cellulose looser and water entrance easier, which also led to higher water absorption by PFOMCF than in SSMCF.

\subsection{Comparisons of flame retardant and smoke suppression}

Flame retardancy and smoke suppression are important characteristics in ensuring the safe use of wood products. Especially in the case of fire, wood products with flame retardancy and smoke suppression characteristics provide more time for rescue and effectively ensure personal 
and property safety [22]. The effects of organic and inorganic impregnation modification on the flame retardancy and smoke suppression properties of Chinese fir wood were evaluated in unmodified wood, PFOMCF, and SSMCF by cone calorimetry. The curves of heat release rate (HRR), total heat release (THR), smoke production rate (SPR), and total smoke production (TSP) are presented in Fig.9.
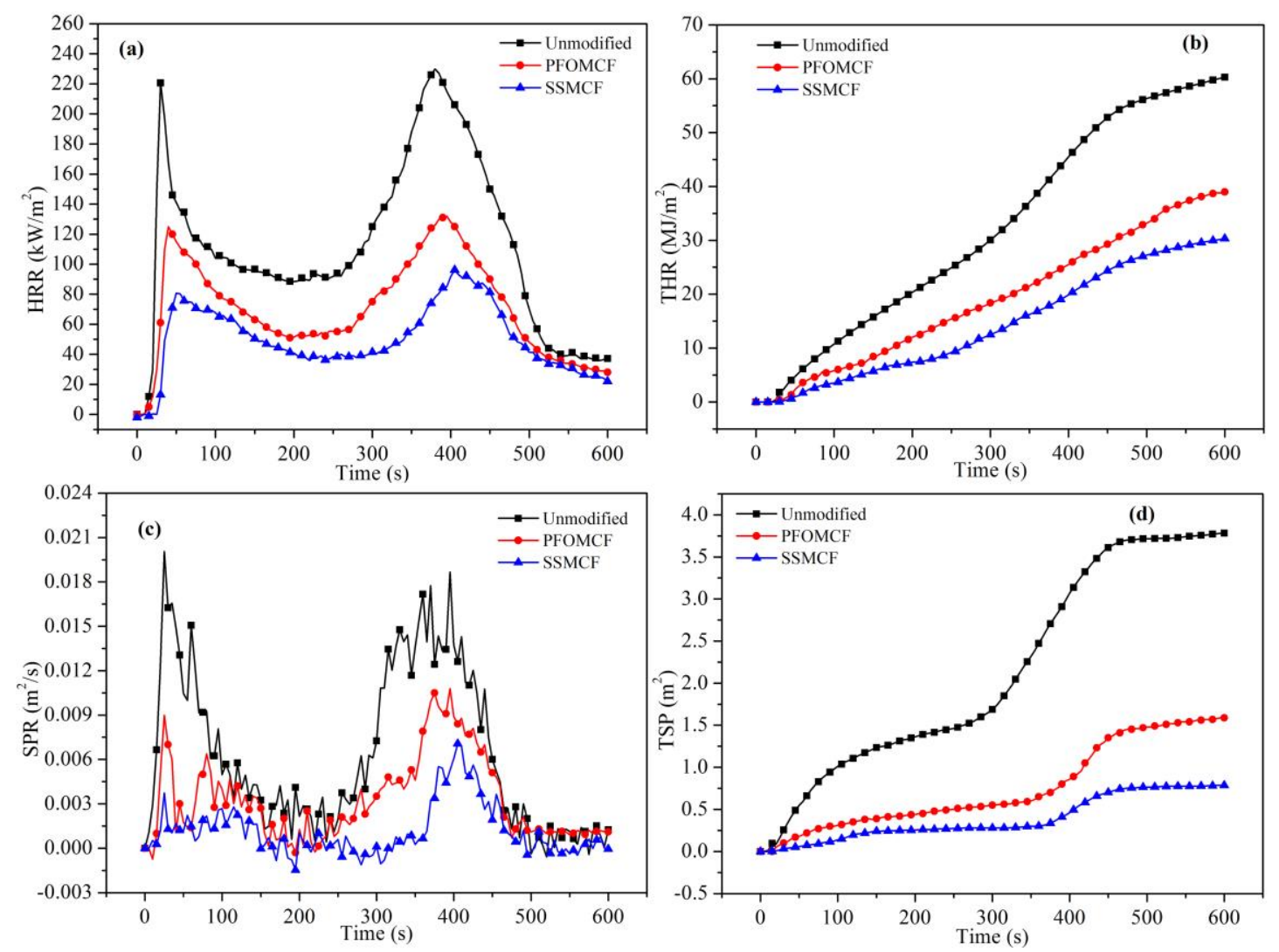

Fig.9 Heat release and smoke formation curves of unmodified Chinese fir wood, PFOMCF and SSMCF

Table1 Heat parameter date of unmodified Chinese fir wood, PFOMCF and SSMCF

\begin{tabular}{cccccc}
\hline Name & $\begin{array}{c}\text { peak-HRR } \\
\left(\mathrm{kW} / \mathrm{m}^{2}\right)\end{array}$ & $\begin{array}{c}\text { mean-HRR } \\
\left(\mathrm{kW} / \mathrm{m}^{2}\right)\end{array}$ & $\begin{array}{c}\text { THR } \\
\left(\mathrm{MJ} / \mathrm{m}^{2}\right)\end{array}$ & $\begin{array}{c}\text { mean-SPR } \\
\left(\mathrm{m}^{2} / \mathrm{s}\right)\end{array}$ & $\begin{array}{c}\text { TSP } \\
\left(\mathrm{m}^{2}\right)\end{array}$ \\
\hline Unmodified & 230.0187 & 32.1838 & 60.2961 & 0.006313 & 3.7821 \\
PFOMCF & 131.2311 & 19.0316 & 39.1132 & 0.003285 & 1.5916 \\
SSMCF & 96.1476 & 14.2745 & 30.4448 & 0.001173 & 0.7873 \\
\hline
\end{tabular}

Fig.9(a) showed that there were two heat release peaks in unmodified wood, PFOMCF, and

SSMCF. The first peak corresponded to the rapid exothermic reaction of wood during decomposition combustion under thermal radiation. At the same time, a carbonized layer formed on the wood surface, which acted as a heat and oxygen insulator. This led to a gentle trough in the HRR curves after the first heat release peak. However, with prolonged combustion time, the wood in the carbonized layer continued to decompose under thermal radiation and high temperature and produced gas products, which burst and cracked the carbonized layer, resulting in rapid decomposition and flammable gas release from the internal wood, thus forming the second combustion heat release peak [23]. Fig.9(a) and (b) and Table1 showed that HRR during the whole 
combustion process showed that the peak heat release rate (peak-HRR), mean heat release rate (mean-HRR), and THR of PFOMCF and SSMCF were lower than those of unmodified wood. This indicated that sodium silicate and phenol formaldehyde oligomer immersion into this wood effectively reduced the wood's HRR and THR. This change was due to impregnating modifiers attached to the wood interior, which helped isolate oxygen and decreased heat transfer, thus protecting the wood and playing a flame-retardant role. Compared with PFOMCF, SSMCF had a lower HRR, peak-HRR, mean-HRR, and THR. On one hand, the flame retardant and smoke suppression performance of inorganic sodium silicate was better than that of organic resin [24]. On the other hand, the weight percentage gain of SSMCF was greater than that of PFOMCF and more flame retardant modifier was thus immersed into the wood.

Smoke and toxic gases in fires were more harmful to human life than fire and heat. Smoke poisoning reduces people's vision and hinders the evacuation and escape of people in a fire, eventually leading to increased suffocation probability. In Fig.9(c), (d) and Table1, the mean-SPR and TSP during the whole combustion time of PFOMCF and SSMCF were significantly lower than those of the unmodified wood. This was because the inorganic sodium silicate and organic phenol formaldehyde oligomer effectively prevented wood from burning, thus reducing the smoke release rate and total smoke production. However, the mean-SRP and TSP of SSMCF was lower than those of PFOMCF, which was due to the better flame retardancy and smoke suppression of inorganic sodium silicate and the larger weight percentage gain of SSMCF.

\subsection{Comparisons of heat resistance}

Under normal conditions, the heat resistance of the inorganic modifier was better than that of the organic modifier. To investigate the effects of the two modifiers on the heat resistance of modified wood, thermogravimetric (TGA) tests were performed on the PFOMCF and SSMCF. The results are shown in Fig.10.
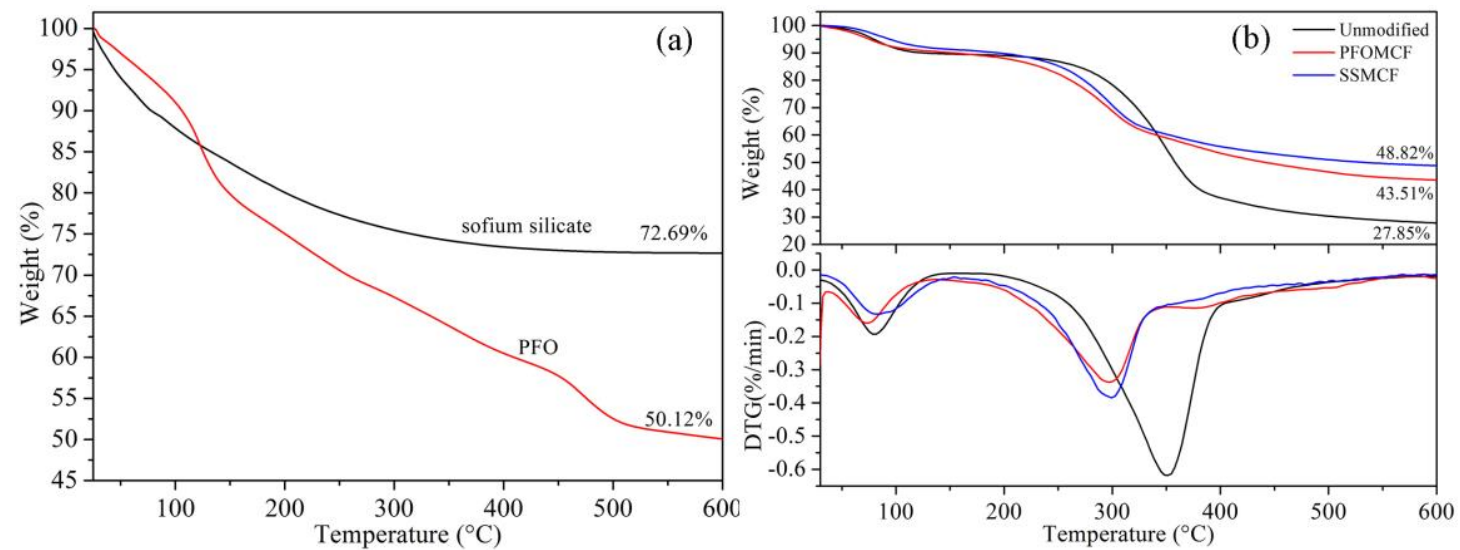

Fig.10 TG-DTG curves of modifiers, unmodified and modified Chinese fir wood

The TGA curves in Fig.10(b) showed that showed that the thermal decomposition rates of PFOMCF and SSMCF were significantly lower than those of unmodified wood at $300^{\circ} \mathrm{C}$. The thermal decomposition residues of PFOMCF and SSMCF increased to 48.82 and $43.51 \%$, respectively (Fig. 10b). The results showed that the heat resistance of modified wood was 
significantly improved after impregnation. However, the initial decomposition temperature and decomposition rates of PFOMCF and SSMCF were slightly lower than those of unmodified wood below $300^{\circ} \mathrm{C}$. At the same time, from DTG curves showed that the maximum decomposition rate temperature of unmodified fir was higher than that of PFOMCF and SSMCF. This was attributed to the observation that the crystalline structure of modified wood was damaged to a certain extent after silicate was introduced into the wood, resulting in amorphous cellulose zones in the wood decomposing more easily with increased heat [25,26]. Comparing the two kinds of impregnated wood in TG and DTG curves, the initial decomposition temperature, maximum decomposition rate temperature, and decomposition residual rate of SSMCF were higher than those of PFOMCF. The results showed that the thermal resistance of wood modified by silicate inorganic impregnation was better than that of phenol formaldehyde oligomer impregnation. The reason for this difference was that sodium silicate had a better thermal stability than phenol formaldehyde oligomer. From Fig.10(a), the decomposition residues of sodium silicate and phenolic oligomers were 72.69 and $50.12 \%$, respectively. When a modifier with good heat resistance was immersed in this wood, the heat resistance was improved. Silicates play an important role in improving the safety of Chinese fir products in case of fire.

\section{Conclusions}

The effects of organic and inorganic impregnation on the properties of Chinese fir wood were compared by modifying the wood with phenol formaldehyde oligomer and sodium silicate using the respiratory impregnation method, forming PFOMCF and SSMCF, respectively. The weight percentage gain, density increase ratio, blending strength, compressive strength, hardness (end face, diameter, and chord face), water absorption rate, chemical structure, crystalline structure, flame retardancy, and heat resistance of PFOMCF and SSMCF were analyzed. The results showed that the impregnation and reinforcement effects and dimensional stability of SSMCF were better than those of PFOMCF. The results of FT-IR and XRD analyses indicated that SSMCF had more hydrogen bonds than PFOMCF and that high bond energy $\mathrm{Si}-\mathrm{O}-\mathrm{Si}$ bonds were formed. Meanwhile, the weaker degree of the SSMCF diffraction peak was much less than that of PFOMCF. These two observations explained that the mechanical properties and water resistance of SSMCF were better than PFOMCF. CONE and TGA results showed that HRR, peak-HRR, mean-HRR, THR, SPR, and TSP of SSMCF were lower than those of PFOMCF, but the thermal decomposition temperature and residual rate were higher. This illustrated that inorganic sodium silicate was a better flame retardant and that SSMCF had a smoke suppression effect as well as better thermal stability and safety performance in case of fire.

\section{Acknowledgments}

This work was financially supported by the Key Laboratory of Bio-based Material Science \& Technology (Northeast Forestry University), Ministry of Education (SWZ-MS201917), Hunan Provincial Technical Innovation Platform and Talent Program in Science and Technology (2019RS2040), Major Science and Technology Program of Hunan Province (2017NK1010), National 
Natural Science Foundation of China (31770606), Scientific Research Project of Hunan Provincial Education Department (17C1500) and Hunan Province Innovation Foundation for Postgraduate (CX20190600).

\section{References}

[1] Chen, G.S.; Yang, Z.J.; Gao, R.; Xie, J.S.; Guo, J.F.; Huang, Z.Q.; Yang, Y.S. Carbon storage in a chronosequence of Chinese fir plantations in southern China. Forest Ecol. Manag. 2013, 300, 68-76.

[2] Duan, H.J.; Cao, S.; Zheng, H.Q.; Hu, D.H.; Lin, J.; Lin, H.Z.; Hu, R.Y.; Sun, Y.S.; Li, Y. Variation in the growth traits and wood properties of Chinese fir from six provinces of southern China. Forests, 2016, 7(9), 192.

[3] Li, P.; Zuo, Y.F.; Wu, Y.Q.; Zhao, X. Study on modification of Chinese fir for furniture and floor by resin impregnation. J. Forest. Eng. 2016,1(5), 133-138.

[4] Yue, K.; Chen, Z.J.; Lu, W.D.; Liu, W.Q.; Li, M.Y.; Shao, Y.L.; Tang, L.J.; Wan, L. Evaluating the mechanical and fire-resistance properties of modified fast-growing Chinese fir timber with boric-phenol-formaldehyde resin. Constr. Build. Mater. 2017, 154, 956-962.

[5] Devi, R.R.; Maji, T.K. Chemical modification of simul wood with styrene-acrylonitrile copolymer and organically modified nanoclay. Wood Sci. Technol. 2012, 46(1-3), 299-315.

[6] Xue, F.L.; Zhao, G.J. Optimum preparation technology for Chinese fir wood/Ca-montmorillonite (Ca-MMT) composite board. Forest. Stud. China. 2008, 10(3), 199.

[7] Yao, M.M.; Yang, Y.Q.; Song, J.L.; Yu, Y.; Jin, Y.C. Melamine formaldehyde modified furfurylation to improve Chinese fir's dimensional stability and mechanical properties. BioResources, 2017, 12(2), 3057-3066.

[8] He, G.B.; Riedl, B.; Ait-Kadi, A. Curing process of powdered phenol-formaldehyde resol resins and the role of water in the curing system. J. Appl. Polym. Sci. 2003, 89(5), 1371-1378.

[9] Shi, J.S.; Li, J.Z.; Zhou, W.R.; Zhang, D.R. Improvement of wood properties by urea-formaldehyde resin and nano-SiO 2 . Front. Forest. China. 2007, 2(1), 104.

[10] Lee, W.J.; Lan, W.C. Properties of resorcinol-tannin-formaldehyde copolymer resins prepared from the bark extracts of Taiwan acacia and China fir. Bioresource Technol. 2006, $97(2), 257-264$.

[11] Mai, C.; Militz, H. Modification of wood with silicon compounds. Inorganic silicon compounds and sol-gel systems: a review. Wood Sci. Technol. 2004, 37(5), 339-348.

[12] Lu, Y.; Feng, M.; Zhan, H.B. Preparation of $\mathrm{SiO}_{2}-$ wood composites by an ultrasonic-assisted sol-gel technique. Cellulose, 2014, 21(6), 4393-4403.

[13] Croitoru, C.; Patachia, S.; Lunguleasa, A. New method of wood impregnation with inorganic compounds using ethyl methylimidazolium chloride as carrier. J. Wood Chem. Technol. 2015, 35(2), 113-128.

[14] Wang, W.B.; Zhao, Z.Y.; Gao, Z.H.; Guo, M.R. Water-resistant whey protein based wood adhesive modified by post-treated phenol-formaldehyde oligomers (PFO). Bioresources, 
2012, 7(2), 1972-1983.

[15] Segal, L.; Creely, J.J.; Martin Jr, A.E.; Conrad, C.M. An empirical method for estimating the degree of crystallinity of native cellulose using the X-ray diffractometer. Textile Research Journal, 1959, 29(10), 786-794.

[16] Yao, M.M.; Yang, Y.Q.; Song, J.L.; Yu, Y.; Jin, Y.C. Lignin-based catalysts for Chinese fir furfurylation to improve dimensional stability and mechanical properties. Ind. Crop. Prod. 2017, 107, 38-44.

[17] Huang, Y.H.; Fei, B.H.; Yu, Y.; Zhao, R.J. Effect of modification with phenol formaldehyde resin on the mechanical properties of wood from Chinese fir. BioResources, 2012, 8(1), $272-282$.

[18] Pérez, J.; Munoz-Dorado, J.; De la Rubia, T.; Martínez, J. Biodegradation and biological treatments of cellulose, hemicellulose and lignin: an overview. Int. Microbiol. 2002, 5(2), 53-63.

[19] Alexandre, M.; Dubois, P. Polymer-layered silicate nanocomposites: preparation, properties and uses of a new class of materials. Mat. Sci. Eng. R. 2000, 28(1-2), 1-63.

[20] Poletto, M.; Zattera, A.J.; Forte, M.M.C.; Santana, R.M.C. Thermal decomposition of wood: Influence of wood components and cellulose crystallite size. Bioresource Technol. 2012, 109, 148-153.

[21] Toba, K.; Yamamoto, H.; Yoshida, M. Crystallization of cellulose microfibrils in wood cell wall by repeated dry-and-wet treatment, using X-ray diffraction technique. Cellulose, 2013, 20(2), 633-643.

[22] Guo, B.T.; Liu, Y.Z.; Zhang, Q.; Wang, F.Q.; Wang, Q.W.; Liu, Y.X.; Li, J.; Yu, H.P. Efficient flame-retardant and smoke-suppression properties of $\mathrm{Mg}$-Al-layered double-hydroxide nanostructures on wood substrate. ACS appl. Mater. Inter. 2017, 9(27), 23039-23047.

[23] Yuan, L.P.; Chen, X.; Hu, Y.C. Combination effect of 4-picolinic acid with 5A zeolite on ammonium polyphosphate flame-retarded sawdust board. J. Fire Sci. 2014, 32(3), 230-240.

[24] Chen, H.; Lang, Q.; Bi, Z.; Miao, X.; Li, Y.; Pu, J. Impregnation of poplar wood (Populus euramericana) with methylolurea and sodium silicate sol and induction of in-situ gel polymerization by heating. Holzforschung, 2014, 68(1), 45-52.

[25] Esteves, B.; Pereira, H. Wood modification by heat treatment: a review. BioResources, 2008, $4(1), 370-404$.

[26] Aydemir, D.; Kiziltas, A.; Kiziltas, E.E.; Gardner, D.J.; Gunduz, G. Heat treated wood-nylon 6 composites. Compos. Part B-Eng. 2015, 68, 414-423 